\title{
ON STOCHASTIC SCHRÖDINGER EQUATION AS A DIRAC BOUNDARY-VALUE PROBLEM, AND AN INDUCTIVE STOCHASTIC LIMIT.
}

\author{
V P BELAVKIN \\ Dedicated to Sergio Albeverio.
}

\begin{abstract}
We prove that a single-jump quantum stochastic unitary evolution is equivalent to a Dirac boundary value problem on the half line in one extra dimension. It is shown that this exactly solvable model can be obtained from a Schrödinger boundary value problem for a positive relativistic Hamiltonian in the half-line as the inductive ultrarelativistic limit, correspondent to the input flow of Dirac particles with asymptotically infinite momenta. Thus the problem of stochastic approximation is reduced to the to the quantum-mechanical boundary value problem in the extra dimension. The question of microscopic time reversibility is also studied for this paper.
\end{abstract}

\section{INTRODUCTION.}

The stochastic evolution models in a Hilbert space have recently found interesting applications in quantum measurement theory, see for example the review paper [1]. In this paper we are going to show on a simple example that classical discontinuous stochastics can be derived from a quantum continuous deterministic conservative dynamics starting from a pure quantum state. It has been already proved in [2] that the piecewise continuous stochastic unitary evolution driven by a quantum Poisson process is equivalent to a time-dependent singular Hamiltonian Schrödinger problem, and the continuous stochastic unitary evolution driven by a quantum Wiener process can be obtained as the solution of this problem at a central limit. Here we want to start form a non-singular time-independent dynamics.

There exits a broad literature on the stochastic limit in quantum physics in which quantum stochastics is derived from a nonsingular interaction representation of the Schrödinger initial value problem for a quantum field by rescaling the time and space as suggested in [3]. Our intention is rather different: instead of rescaling the interaction potentials we treat the singular interactions rigorously as the boundary conditions, and obtain the stochastic limit as an ultrarelativistic limit of the corresponding Schrödinger boundary value problem in a Hilbert space of infinite number of particles. We shall prove that the discontinuous and continuous quantum stochastic evolutions can be obtain in this way from a physically meaningful

Date: January 12, 2000.

Key words and phrases. Quantum probability, Boundary value problem, Dirac equation, Stochastic approximation.

Published in: Evolution Equations and Their Appications, Marcel Dekker, Inc: New York 2000, pp. 311-327.

This work was supported by Royal Society grant for UK-Japan collaboration and EPSRC research grant GR/M66196. 
time continuous (in strong sense) unitary evolution by solving a boundary value problem with an initial pure state in the extended Hilbert space.

First we shall describe the boundary value problem corresponding to the singlepoint discontinuous stochastic evolution and demonstrate the ultrarelativistic limit in this case. Then the piece-wise continuous stochastic evolution and the continuous diffusive and quantum stochastic evolution can be obtained as in [2] 4]. But before to perform this program, let us describe the unitary toy model giving an "unphysical" solution of this problem corresponding to the free hamiltonian $h(p)=-p$. This toy model in the second quantization framework was suggested for the derivation of quantum time-continuous measurement process in [5]. Recently Chebotarev [6] has shown that the secondary quantized time-continuous toy Hamiltonian model in Fock space with a discontinuity condition is equivalent to the Hudson-Parthasarathy (HP) quantum stochastic evolution model [7] in the case of commuting operator-valued coefficients of the HP-equation. Our approach is free from the commutativity restriction for the coefficients, and we deal with timereversible Dirac Hamiltonian and the boundary rather than physically meaningless discontinuity condition and time irreversible $-p$. Moreover, we shall prove that the stochastic model can be obtained from a positive relativistic Hamiltonian as an inductive ultra relativistic limit on a union of Hardy class Hilbert spaces. We call this limit the inductive stochastic approximation.

\section{A toy Hamiltonian model.}

Here we demonstrate on a toy model how the time-dependent single-point stochastic Hamiltonian problem can be treated as an interaction representation of a self-adjoint boundary-value Schrödinger problem for a strongly-continuous unitary group evolution.

Let $\mathcal{H}$ be a Hilbert space, $H$ be a bounded from below selfadjoint operator, and $S$ be a unitary operator in $\mathcal{H}$, not necessarily commuting with $H$. The operator $H$ called Hamiltonian, is the generator for the conservative evolution of a quantum system, described by the Schrödinger equation $i \hbar \partial_{t} \eta=H \eta$, and the operator $S$ called scattering, describes the unitary quantum jump $\eta \mapsto S \eta$ of the state vectors $\eta \in \mathcal{H}$ caused by a singular potential interaction in the system, with the continuous unitary evolution $\eta \mapsto e^{-\frac{i}{\hbar} t H} \eta$ when there is no jump. As for an example of such jump we can refer to the von Neumann singular Hamiltonian model for indirect instantaneous measurement of a quantum particle position $x \in \mathbb{R}$ via the registration of an apparatus pointer position $y \in \mathbb{R}$. It can be described 2 , 4 by the $x$-pointwise shift $S$ of $y$ as the multiplication $\hat{\sigma}$ by $\sigma(x)=e^{x \partial_{y}}$ in the Hilbert space $L^{2}\left(\mathbb{R}^{2}\right)$ of square-integrable functions $\eta(x, y)$, and it does not commute with the free Hamiltonian operator $H=\frac{1}{2}\left(y^{2}-\partial_{x}^{2}\right)$ say, of the system "quantum particle plus apparatus pointer".

It is usually assumed that the quantum jump occurs at a random instant of time $t=s$ with a given probability density $\rho(s)>0$ on the positive half of line $\mathbb{R}^{+}$, $\int_{0}^{\infty} \rho(s) \mathrm{d} s=1$. If $H$ and $S$ commute, the single-point discontinuous in $t$ stochastic evolution can formally be described by the time-dependent Schrödinger equation $i \hbar \partial_{t} \chi(t)=H_{s}(t) \chi(t)$ with the singular stochastic Hamiltonian

$$
H_{s}(t)=H+i \hbar \delta_{s}(t) \ln S,
$$


where $\delta_{s}(t)=\delta(t-s)=\delta_{t}(s)$ is the Dirac $\delta$-function of $z=s-t$. Indeed, integrating the time-dependent Hamiltonian $H_{r}(s)$ over $r$ from 0 to $t$ for a fixed $s \in \mathbb{R}$, one can obtain

$$
V(t, s)=e^{-\frac{i}{\hbar} \int_{0}^{t} H_{s}(r) \mathrm{d} r}=e^{-\frac{i}{\hbar} t H} S^{\Delta_{0}^{t}(s)}=e^{\frac{i}{\hbar}(s-t) H} S^{\Delta_{0}^{t}(s)} e^{-\frac{i}{\hbar} s H},
$$

where $\Delta_{0}^{t}(s)=\int_{0}^{t} \delta_{r}(s) \mathrm{d} r$ is identified with the indicator function $1_{[0, t)}$ of the interval $[0, t)$ for a $t>0$ (at $t \leq 0$ it is zero if $s>0$ ). The right hand side is the form of the unitary stochastic evolution $V(t, s)$ which should remain valid even if the operators $H$ and $S$ do not commute. First the evolution is conservative and continuous, $V(t, s)=e^{-\frac{i}{\hbar} t H}$ for $t \in[0, s)$, then the quantum jump $S$ is applied at $t=s$, and at $t>s$ the evolution is again continuous, described by the Hamiltonian $H$. As it was noted in [2], the rigorous form of the stochastic Schrödinger equation which gives such solution even for noncommuting $H$ and $S$ in the positive direction of $t$, is the Ito differential equation

$$
\mathrm{d}_{t} V(t, s)+\frac{i}{\hbar} H V(t, s) \mathrm{d} t=(S-I) V(t, s) \mathrm{d} 1_{t}(s), t>0, \quad V(0, s)=I .
$$

Here $\mathrm{d}_{t} V(t, s)=V(t+\mathrm{d} t, s)-V(t, s)$ is the forward differential corresponding to an infinitesimal increment $\mathrm{d} t>0$ at $t$, and $\mathrm{d} 1_{t}(s)=\Delta_{0}^{\mathrm{d} t}(s-t)$ is the indicator function $\Delta_{t}^{\mathrm{d} t}(s)=1_{[t, t+\mathrm{d} t)}(s)$, the forward increment of the Heaviside function $t \mapsto 1_{t}(s)=1_{0}(s-t)$, where $1_{0}=1_{(-\infty, 0)}$. The equation (2.2) simply means that $t \mapsto V(t)$ for a fixed $s=z$ satisfies the usual Schrödinger equation $i \hbar \partial_{t} V(t)=$ $H V(t)$ if $t \neq s$ as $\mathrm{d} 1_{t}(s)=0$ for a sufficiently small $\mathrm{d} t(\mathrm{~d} t<s-t$ if $t<s$, and any $\mathrm{d} t>0$ if $t>s)$, while it jumps, $\mathrm{d}_{t} V=(S-I) V$ at $t=s$ as $\left.\mathrm{d} 1_{t}(s)\right|_{t=s}=1 \gg \mathrm{d} t$. Integrating $\mathrm{d}_{z} \chi(z)=\mathrm{d}_{z} V(z) \eta$ on the domain of the operator $H$ first from 0 to $z=s$ with an initial condition $\chi(0)=\eta$, and then from $s$ to $t$ with the initial condition $\chi\left(s_{+}\right):=\lim _{z \searrow s} \chi(z)=S \chi(s)$ one can easily obtain the solution in the form $\chi(t, s)=V(t, s) \eta$, where

$$
V(t, z)=e^{-\frac{i}{\hbar} t H} S(z)^{1_{[0, t)}(z)}, \quad S(z)=e^{\frac{i}{\hbar} z H} S e^{-\frac{i}{\hbar} z H}
$$

without the commutativity condition for $H$ and $S$.

Now we shall prove that the stochstic single-jump discontinuous evolution $V(t)$ can be treated as the interaction representation $V(t) \chi^{0}=e^{\frac{i}{\hbar} t h(\hat{p})} \chi^{t}$ for a deterministic strongly-continuous Schrödinger evolution $\chi^{0} \mapsto \chi^{t}$ in one extra dimension $z \in \mathbb{R}$ with the initial conditions $\chi^{0}(z)=\sqrt{\rho(z)} \eta \in \mathcal{H}$ localized at $z>0$ : $\chi^{0}(z)=0$ at $z \leq 0$. Here $h(\hat{p})=-\hat{p}$ is the free Hamiltonian, where $\hat{p}=-i \hbar \partial_{z}$ is the momentum in one extra dimension of $z \in \mathbb{R}$.

Proposition 1. The described stochastic Hamiltonian problem 2.2) is unitary equivalent to the self-adjoint boundary-value Schrödinger problem

$$
i \hbar \partial_{t} \chi^{t}(z)=\left(H+i \hbar \partial_{z}\right) \chi^{t}(z), \quad \chi^{t}\left(0_{-}\right)=S \chi^{t}(0)
$$

in the Hilbert space $\mathcal{H} \otimes L^{2}(\mathbb{R})$ in the sense that the stochastic evolution $V(t)$ at $t>0$ coincides with the unitary cocycle $V(t, z) \chi^{0}(z)=\chi^{t}(z-t)$ resolving the boundary value problem (2.4) with respect to the plane propagation $e^{t \partial_{z}}$ along $z$ as $\chi^{t}=e^{t \partial_{z}} V(t) \chi^{0}, \forall \chi^{0} \in \mathcal{H} \otimes L^{2}(\mathbb{R})$.

Proof. The boundary value problem (2.4) is well defined on the space of smooth square-integrable $\mathcal{H}$-valued functions $\chi$, and is symmetric as $H$ is self-adjoint, and 
due to the unitary boundary condition

$$
0=\left(\|\chi(-0)\|^{2}-\|\chi(0)\|^{2}\right)=2 \int \operatorname{Re}\left\langle\chi(z) \mid \chi^{\prime}(z)\right\rangle \mathrm{d} z=\frac{2}{\hbar} \operatorname{Im}\langle\chi \mid \hat{h} \chi\rangle .
$$

In fact, this problem is selfadjoint as it has apparently unitary solution

$$
\chi^{t}(z)=e^{\frac{i}{\hbar} z H} \chi_{t}(z+t), \quad \chi_{t}(s)=S^{\Delta_{0}^{t}(s)} \chi_{0}(s),
$$

where $\chi_{0}(s)=e^{-\frac{i}{\hbar} H z} \chi^{0}(s)$. Indeed, substituting $\chi^{t}(z)=e^{\frac{i}{\hbar} z H} \chi_{0}^{t}(z)$ into the equation $\{2.4\}$ we obtain the transport equation $\partial_{t} \chi_{0}^{t}(z)=\partial_{z} \chi_{0}^{t}(z)$ with the same boundary condition $\chi_{0}^{t}(-0)=S \chi_{0}^{t}(0)$ and the initial condition $\chi_{0}^{0}=\chi_{0}$ corresponding to a $\chi^{0} \in \mathcal{H} \otimes L^{2}(\mathbb{R})$. This simple initial boundary-value problem has the obvious solution $\chi_{0}^{t}(z)=\chi_{t}(z+t)$ with $\chi_{t}$ given in (2.5) as

$$
\chi_{t}(s)=S^{1_{(0, t]}(t-s)} \chi^{0}(s), t>0, \quad \chi_{t}(s)=S^{-1_{[-t, 0)}(s)} \chi^{0}(s), t<0 .
$$

The unitarity of $S^{\Delta_{0}^{t}(s)}$ in $\mathcal{H}$ and of shift $e^{t \partial_{z}}$ in $L^{2}(\mathbb{R})$ implies the unitarity of the resolving map $V^{t}: \chi^{0} \mapsto \chi^{t}$ in $\mathcal{H} \otimes L^{2}(\mathbb{R})$,

$$
\left\|\chi^{t}\right\|^{2}=\left\|\chi_{t}\right\|^{2}=\left\|\chi_{0}\right\|^{2}=\left\|\chi^{0}\right\|^{2}
$$

Moreover, the map $t \mapsto V^{t}$ has the multiplicative representation property $V^{r} V^{t}=$ $V^{r+t}$ of the group $\mathbb{R} \ni r, t$ because the map $t \mapsto S^{\Delta_{0}^{t}(s)}$ is a multiplicative shiftcocycle,

$$
S^{\Delta_{0}^{r}(s)} e^{t \partial_{s}} S^{\Delta_{0}^{t}(s)}=e^{t \partial_{s}} S^{\Delta_{0}^{r+t}(s)}, \quad \forall r, t \in \mathbb{R}
$$

by virtue of the additive cocycle property for the commuting $\Delta_{0}^{t}(s)=1_{t}(s)-1_{0}(s)$ :

$$
\left[\Delta_{0}^{r}+e^{t \partial_{s}} \Delta_{0}^{t}\right](s)=1_{r}(s)-1_{0}(s)+1_{t}(s+t)-1_{0}(s+t)=e^{t \partial_{s}} \Delta_{0}^{r+t}(s) .
$$

The subtraction $\chi(t, z)=e^{\frac{i}{\hbar} t \hat{h}} \chi^{t}(z)$ of free evolution with the generator $\hat{h} \chi(z)=$ $i \hbar \partial_{z} \chi(z)$ obviously gives

$$
\begin{aligned}
\chi(t, s) & =\chi^{t}(s-t)=e^{\frac{i}{\hbar}(s-t) H} \chi_{t}(s) \\
& =e^{\frac{i}{\hbar}(s-t) H} S^{\Delta_{0}^{t}(s)} e^{-\frac{i}{\hbar} s H} \chi^{0}=V(t, s) \chi^{0}(s),
\end{aligned}
$$

Thus the single-point discontinuous unitary $e^{-\frac{i}{\hbar} t \hat{h}}$-cocycle

$$
V(t, s)=e^{\frac{i}{\hbar} t \hat{h}} V^{t}=e^{\frac{i}{\hbar}(s-t) H} S^{\Delta_{0}^{t}(s)} e^{-\frac{i}{\hbar} s H}, \quad t \in \mathbb{R}
$$

with $\Delta_{0}^{t}(s)=1_{[0, t)}(s)$ for a positive $t$ and $s \in \mathbb{R}^{+}$, solves indeed the single-jump Ito equation (2.2). It describes the interaction representation for the strongly continuous unitary group evolution $V^{t}$ resolving the boundary value problem (2.4) with initially constant functions $\chi^{0}(s)=\eta$ at $s>0$.

Remark 1. The toy Schrödinger boundary value problem 2.4) is unphysical in three aspects. First, the equation 2.4 is not invariant under the reversion of time arrow, i.e. under an isometric complex conjugation $\eta \mapsto \bar{\eta}$ and the reflection $t \mapsto-t$, even if $\bar{S}=S^{-1}$ and $\operatorname{Im} H=0$ as the Hamiltonian $\hat{h}=i \hbar \partial_{z}$ is not real, $\operatorname{Im} \hat{h}=\hbar \partial_{z}$. Second, a physical wave function $\psi^{t}(z)$ should have a continuous propagation in both directions of $z$, and at the boundary must have a jump not in the coordinate but in momentum representation. The momentum can change its direction but not the magnitude (conservation of momentum) in the result of the singular interaction with the boundary. And third, the free Hamiltonian $\hat{h}$ must be bounded from below which is not so in the case of hamiltonian function $h(z, p)=-p$ corresponding to the equation (2.4). 
Now we show how to rectify the first two failures of the toy model, but the third, which is a more serious failure, will be sorted out in the next sections by considering the toy model as a dressed limiting case.

Instead of the single wave function $\chi^{t}(z)$ on $\mathbb{R}$ let us considering the pair $(\psi, \tilde{\psi})$ of input and output wave functions with

$$
\psi^{t}(z)=\chi^{t}(z), z \geq 0, \quad \tilde{\psi}^{t}(-z)=\chi^{t}(z), z<0
$$

on the half of line $\mathbb{R}^{+}$, having the scalar product

$$
\int_{0}^{\infty}\left(\|\psi(z)\|^{2}+\|\tilde{\psi}(z)\|^{2}\right) \rho(z) \mathrm{d} z=\int_{-\infty}^{\infty}\|\chi(z)\|^{2} \rho(z) \mathrm{d} z .
$$

They satisfy the system of equations

$$
\begin{array}{ll}
i \hbar \partial_{t} \psi^{t}(z)=\left(H+i \hbar \partial_{z}\right) \psi^{t}(z), & \psi^{0} \in \mathcal{H} \otimes L^{2}\left(\mathbb{R}^{+}\right) \\
i \hbar \partial_{t} \tilde{\psi}^{t}(z)=\left(H-i \hbar \partial_{z}\right) \tilde{\psi}^{t}(z), & \tilde{\psi}^{0} \in \mathcal{H} \otimes L^{2}\left(\mathbb{R}^{+}\right)
\end{array}
$$

for a quantum system interacting with a massless Dirac particle in $\mathbb{R}^{+}$through the boundary condition $\tilde{\psi}^{t}(0)=S \psi^{t}(0)$, where $\tilde{\psi}^{t}(0)=\chi^{t}\left(0_{-}\right)$. One can show that this is indeed the diagonal form of the Dirac equation in one dimension in the eigen-representation of the Dirac velocity $c=-\sigma_{z}$ along $z \in \mathbb{R}^{+}$, with the electric and magnetic field components $u_{ \pm}$, given by the symmetric and atisymmetric parts $u \pm \tilde{u}$ of $u$ on $\mathbb{R}$ in the case $\operatorname{Im} u=0$. The components of $(\psi, \tilde{\psi})$ propagate independently at $z>0$ as plane waves in the opposite directions with a spin (or polarization) oriented in the direction of $z$, and in the scalar case $\mathcal{H}=\mathbb{C}$ are connected by the Dirac type boundary condition $(1+i \mu) \tilde{\psi}^{t}(0)=(1-i \mu) \psi^{t}(0)$ correspondent to a point mass $\hbar \mu$ at $z=0$. The input wave function

$$
\psi^{t}(z)=e^{-\frac{i}{\hbar} t H} \psi^{0}(z+t)=e^{-\frac{i}{\hbar} t(\hat{h}+H)} \psi^{0}(z)
$$

is the solution to the equation (2.4) at $z \in \mathbb{R}^{+}$with $\left.\chi^{0}\right|_{z>0}=\psi^{0}$ which does not need the boundary condition at $z=0$ when solving the Cauchy problem in $t>0$. The output wave function satisfies the reflected equation at $z>0$ and the unitary boundary condition at $z=0$ :

$$
i \hbar \partial_{t} \tilde{\psi}^{t}(z)=\left(H-i \hbar \partial_{z}\right) \tilde{\psi}^{t}(z), \quad \tilde{\psi}^{t}(0)=S \psi^{t}(0)
$$

It has the solution

$$
\tilde{\psi}^{t}(z)=e^{-\frac{i}{\hbar} t H}\left[\tilde{\psi}^{0}(z-t) 1_{t}^{\perp}(z)+S(t-z) \psi^{0}(t-z) 1_{t}(z)\right],
$$

where $1_{t}^{\perp}(z)=1-1_{t}(z)$. This can be written in the similar way as $\psi^{t}$,

$$
\tilde{\psi}^{t}(z)=e^{-\frac{i}{\hbar} t H} \tilde{\psi}^{0}(z-t)=e^{-\frac{i}{\hbar} t(\check{h}+H)} \tilde{\psi}^{0}(z)
$$

with $\check{h}=-i \hbar \partial_{z}$ if $\psi^{0}(z)$ is extended into the domain $z<0$ as

$$
S(z) \psi^{t}(z)=\tilde{\psi}^{t}(-z), \quad S(z)=e^{\frac{i}{\hbar} z H} S e^{-\frac{i}{\hbar} z H}
$$

at $t=0$. Note that reflection condition (2.10) remains valid for all $t>0$ if $\psi^{t}$ is extended into the region $z<0$ by (2.7) for all $t \in \mathbb{R}^{+}$:

$$
\tilde{\psi}^{t}(-z)=e^{-\frac{i}{\hbar} t H} S(t+z) \psi^{0}(t+z)=S(z) e^{-\frac{i}{\hbar} t H} \psi^{0}(z+t)=S(z) \psi^{t}(z) .
$$


Extending also the output wave $\tilde{\psi}^{t}$ by (2.9) into the region $z<0$ we obtain the continuous propagation of $\psi, \tilde{\psi}$ through the boundary in the opposite directions, with the unitary reflection holonome connection (2.10) for all $z \in \mathbb{R}$. If $\bar{H}=H$, where $\bar{H} \eta=\bar{H} \bar{\eta}$ with respect to a complex conjugation in $\mathcal{H}$, then the system of Schrödinger equations for the pair $(\psi, \tilde{\psi})$ remains invariant under the time reflection with complex conjugation up to exchange $\bar{\psi}^{-t} \rightleftarrows \tilde{\psi}^{t}$. Indeed, in this case the complex conjugated hamiltonian $\overline{\hat{h}}=-i \hbar \partial_{z}$ coinsides with the operator $\check{h}$ corresponding to $\tilde{h}(z, p)=p=\bar{h}(z, p)$. The boundary value problem is invariant under time reversion if $\bar{S}=S^{-1}$ as the reflection condition (2.10) is extended to the negative $t$ by the exchange due to $S(z)^{-1}=\bar{S}(-z)$. Thus the reversion of time arrow is equivalent to the exchange of the input and output wave functions which is an involute isomorphism due to

$$
\int_{-\infty}^{\infty}\|\psi(z)\|^{2} \mathrm{~d} z=\|\chi\|^{2}=\int_{-\infty}^{\infty}\|\tilde{\psi}(z)\|^{2} \mathrm{~d} z .
$$

\section{A UNitARY REFLECTION MODEL}

As we have seen in the end of the previous section, a unitary quantum state jump at a random instant of time $s \geq 0$ is a result of solving of the toy Schrödinger boundary value problem in the interaction representation for a strongly continuous unitary evolution of a Dirac particle with zero mass. The input particle, an "instanton" with the state vectors defining the input probabilities for $s=z$, has the unbounded from below kinetic energy $e(p)=-p$ corresponding to the constant negative velocity $v=e^{\prime}(p)=-1$ along the intrinsic time coordinate $z$ which does not coincide with the direction of the momentum if $p>0$. One can interpret such strange particle as a trigger for instantaneous measurement in a quantum system at the time $z \in \mathbb{R}^{+}$, and might like to consider it as a normal particle, like a "bubble" in a cloud chamber on the boundary of $\mathbb{R}^{d} \times \mathbb{R}^{+}$as it was assumed in 2], with positive kinetic energy and a non-zero mass.

Our aim is to obtain the instanton as an ultrarelativistic limit of a quantum particle with a positive kinetic energy corresponding to a mass $m_{0} \geq 0$. Here we shall treat the kinetic energy separately for input and output instantons as a function of the momentum $p \in \mathbb{R}^{-}$and $p \in \mathbb{R}^{+}$respectively along a coordinate $z \in \mathbb{R}^{+}$with the same self-adjoint operator values $e(p) \geq 0$ in a Hilbert space $\mathfrak{h}$ of its spin or other degrees of freedom. For example one can take the relativistic mass operator-function

$$
e(p)=\left(p^{2}+\hbar^{2} \mu^{2}\right)^{1 / 2}, \quad \mu^{2}=\mu_{0}^{2}-\nabla^{2}
$$

in the Hilbert space $\mathfrak{h}=L^{2}\left(\mathbb{R}^{d}\right)$ which defines the velocities $v(p)=p / e(p)=e^{\prime}(p)$ with the same signature as $p$. At the boundary $z=0$ the incoming particle with the negative momentum $p<0$ is reflected into the outgoing one with the opposite momentum $-p$. The singular interaction with the boundary causes also a quantum jump in other degrees of freedom. It is described by the unitary operator $\sigma$ in $\mathfrak{h}$ which is assumed to commute with $e(p)$ for each $p$ as it is in the quantum measurement model [2] when $\sigma=e^{\mathbf{x} \partial_{\mathbf{y}}}$ with $\nabla=\partial_{\mathbf{y}}$ in $\{3.1\}$.

Let $\mathfrak{h}$ be a Hilbert space with isometric complex conjugation $\mathfrak{h} \ni \eta \mapsto \bar{\eta} \in \mathfrak{h}$, and $L_{\mathfrak{h}}^{2}\left(\mathbb{R}^{-}\right)=\mathfrak{h} \otimes L^{2}\left(\mathbb{R}^{-}\right)$be the space of square-integrable vector-functions $f(k) \in \mathfrak{h}$ 
on the half-line $\mathbb{R}^{-} \ni k$. We denote by $\mathcal{E}^{-}$the isomorphic space of Fourier integrals

$$
\varphi(z)=\frac{1}{2 \pi} \int_{-\infty}^{0} e^{i k z} f(k) \mathrm{d} k, \quad f \in L_{\mathfrak{h}}^{2}\left(\mathbb{R}^{-}\right) .
$$

which is the Hardy class of $\mathfrak{h}$-valued functions $\varphi \in L_{\mathfrak{h}}^{2}(\mathbb{R})$ having the analytical continuation into the complex domain $\operatorname{Im} z<0$. One can interpret $L_{\mathfrak{h}}^{2}\left(\mathbb{R}^{-}\right)$as the Hilbert space of quantum input states with negative momenta $p_{k}=\hbar k, k<0$ along $z \in \mathbb{R}$ and spin states $\eta \in \mathfrak{h}$. The generalized eigen-functions

$$
\varphi_{k}(z)=\exp [i k z] \eta_{k}, k<0, \quad e(\hbar k) \eta_{k}=\hbar \varepsilon_{k} \eta_{k}
$$

corresponding to spectral values $\varepsilon_{k} \in \mathbb{R}^{+}$of $\varepsilon(k)=\hbar^{-1} e(\hbar k)$, are given as the harmonic waves moving from infinity towards $z=0$ with the phase speed $\varsigma_{k}=$ $\varepsilon_{k} /|k|$ along $z$. The amplitudes $\eta_{k}$ are arbitrary in $\mathfrak{h}$ if all $e(p)$ are proportional to the identity operator 1 in $\mathfrak{h}, \varepsilon(k)=\varepsilon_{k} 1$, as it was in the previous section where $\mathfrak{h}=\mathcal{H}$.

The singular interaction creates the output states in the same region $z>0$ of observation where the input field is, by the momentum inversion $p=-p_{k} \mapsto \tilde{p}=p_{k}$, reflecting the input wave functions $\varphi \in \mathcal{E}^{-}$isometrically onto

$$
\tilde{\varphi}(s)=\frac{1}{2 \pi} \int_{-\infty}^{0} e^{-i k s} \tilde{f}(k) \mathrm{d} k=\sigma \varphi(-s), \quad s \in \mathbb{R}_{+}
$$

by $\tilde{f}(k)=\sigma f(k), k<0$. The space $\mathcal{E}^{+}=\left\{\tilde{\varphi}: \varphi \in \mathcal{E}^{-}\right\}$is the conjugated Hardy subspace $\mathcal{E}^{+}=\left\{\bar{\varphi}: \varphi \in \mathcal{E}^{-}\right\}$of analytical functions $\bar{\varphi}(z)=\overline{\varphi(\bar{z})}$ in $\operatorname{Im} z>0$. The reflected wave function satisfies the boundary condition $\tilde{\varphi}(0)=\sigma \varphi(0)$ corresponding to the zero probability current

$$
j(z)=\|\tilde{\varphi}(z)\|^{2}-\|\varphi(z)\|^{2}
$$

at $z=0$, and together with the input wave function $\varphi(s), s \geq 0$ represents the Hilbert square norms (total probability) in $\mathcal{E}^{-}$and $\mathcal{E}^{+}$by the sum of the integrals over the half-region $\mathbb{R}_{+}$:

$$
\int_{-\infty}^{\infty}\|\varphi(z)\|^{2} \mathrm{~d} z=\int_{0}^{\infty}\left(\|\varphi(s)\|^{2}+\|\widetilde{\varphi}(s)\|^{2}\right) \mathrm{d} s=\int_{-\infty}^{\infty}\|\tilde{\varphi}(z)\|^{2} \mathrm{~d} z .
$$

The free dynamics of the input and output wave functions can be described as the unitary propagation

$$
\begin{aligned}
\varphi^{t}(z) & =\frac{1}{2 \pi} \int_{-\infty}^{0} e^{i k(t \varsigma(k)+z)} f(k) \mathrm{d} k=\left[e^{-i t \hat{\varepsilon}} \varphi\right](z), \\
\widetilde{\varphi}^{t}(z) & =\frac{1}{2 \pi} \int_{-\infty}^{0} e^{i k(t \varsigma(k)-z)} \tilde{f}(k) \mathrm{d} k=\left[e^{-i t \tilde{\varepsilon}} \widetilde{\varphi}\right](z),
\end{aligned}
$$

of a superposition of the harmonoc eigen-functions (3.2) in the negative and positive direction of $z \in \mathbb{R}$ respectively with the same phase speeds $\varsigma_{k}>1$ which are the eigen-values of the positive operators $\varsigma(k)=|k|^{-1} \varepsilon(k)$. The generating selfadjoint operators $\hat{\varepsilon}$, $\check{\varepsilon}$ are the restrictions $\hat{\varepsilon}=\varepsilon\left(i \partial_{z}\right)\left|\mathcal{D}^{-}, \check{\varepsilon}=\varepsilon\left(i \partial_{z}\right)\right| \mathcal{D}^{+}$of the kinetic energy operator given by the symmetric function $\varepsilon(p)$ on its symmetric dense domain $\mathcal{D} \subseteq L_{\mathfrak{h}}^{2}(\mathbb{R})$, to the dense domains $\mathcal{D}^{\mp}=\mathcal{D} \cap \mathcal{E}^{\mp}$ in the invariant subspaces $\mathcal{E}^{\mp} \subseteq L_{\mathfrak{h}}^{2}(\mathbb{R})$. 
Instead of dealing with the free propagation of the input-output pair $(\varphi, \tilde{\varphi})$ at the region $z>0$ with the boundary condition $\tilde{\varphi}^{t}(0)=\sigma \varphi^{t}(0)$, it is convenient to introduce just one wave function

$$
\phi^{t}(z)=\varphi^{t}(z), \operatorname{Re} z \geq 0, \quad \phi^{t}(z)=\tilde{\varphi}^{t}(-z), \operatorname{Re} z<0
$$

considering the reflected wave as propagating in the negative direction into the region $z<0$. Each $\phi(-z)$ is a Hardy class function $\sigma \varphi$ at $z>0$, as well as it is Hardy class function $\varphi(-z)$ at $z>0$, but the continuity of the analytical wave functions $\varphi(z)$ at $\operatorname{Re} z=0$ corresponds to the left discontinuity $\phi\left(0_{-}\right)=\sigma \phi(0)$ of

$$
\phi(z)=1_{0}(-z) \varphi(z)+1_{0}(z) \sigma \varphi(z), \quad 1_{0}(z)=\left\{\begin{array}{l}
0, z \geq 0 \\
1, z<0
\end{array},\right.
$$

where $\phi\left(0_{-}\right)$is defined as the left lower sectorial limit of $\phi(z)$ at $\operatorname{Re} z \nearrow 0, \operatorname{Im} z \nearrow 0$. Obviously the Hilbert subspace $\sigma^{\hat{1}_{0}} \mathcal{E}^{-} \subset L_{\mathfrak{h}}^{2}(\mathbb{R})$ of such wave functions is isomorphic to $\mathcal{E}^{-}$by the unitary operator $\sigma^{\hat{1}_{0}}=I+\hat{1}_{0}(\sigma-I)$, where $\hat{1}_{0}$ is the multiplication operator of $\varphi(z)$ by 1 if $z<0$, and by 0 if $z \geq 0$. The unitary evolution group $v^{t}=\sigma^{\hat{1}_{0}} e^{-i t \hat{\varepsilon}} \sigma^{-\hat{1}_{0}}, t \in \mathbb{R}$ for

$$
\phi^{t}(z)=\varphi^{t}(z)+1_{0}(z)(\sigma-1) \varphi^{t}(z)=\sigma^{1_{0}(z)} \varphi^{t}(z),
$$

is unitary equivalent but different from the free propagation $e^{-i t \hat{\varepsilon}}$ of $\varphi^{t}$ in $\mathbb{R}$. Each harmonic eigen-function (3.2) having the plane wave propagation

$$
\varphi_{k}^{t}(z)=e^{-i \varepsilon_{k} t} \varphi_{k}(z)=\varphi_{k}\left(z+\varsigma_{k} t\right),
$$

for the negative $k \in \mathbb{R}^{-}$, is now truncated, $\phi_{k}(z)=e^{i k z} \sigma^{1_{0}(z)} \eta_{k}$, and propagates in the negative direction as

$$
\phi_{k}^{t}(z)=\sigma^{1_{0}(z)} \varphi_{k}\left(z+\varsigma_{k} t\right)=e^{-i \varepsilon_{k} t} \phi_{k}(z) \neq \phi_{k}\left(z+\varsigma_{k} t\right),
$$

keeping the truncation at $z=0$. Therefore the subtraction $\phi_{t}(z)=e^{i t \hat{\varepsilon}} \phi^{t}(z)$ of the free propagation of $\varphi^{t}$ from $\phi^{t}$ does not return it to the initial $\phi^{0}=\sigma^{\hat{1}_{0}} \varphi^{0}$ but to $\phi_{t}=\sigma^{\hat{\pi}^{t}} \varphi^{0}=v_{t} \phi_{0}$, where $\hat{\pi}^{t}=e^{i t \hat{\varepsilon}} \hat{1}_{0} e^{-i t \hat{\varepsilon}}, v_{t}=\sigma^{\hat{\pi}^{t}} \sigma^{-\hat{1}_{0}}$, and $\phi_{0}=\phi^{0}$. Thus we have proved the following proposition for the particular case $\varkappa=0$ of an operator $\varkappa \in \mathbb{R}$, defined in the Proposition 1 of previous section as $\hbar \varkappa=H$ in $\mathfrak{h}=\mathcal{H}$.

Let $\varkappa$ be a selfajoint operator in $\mathfrak{h}$, and $\epsilon_{\varkappa}(z)=e^{-i \varkappa z}$ be the correspondent oneparameter unitary group in $\mathfrak{h}$. Below we shall denote by $\hat{\epsilon}_{\varkappa}$ and $\check{\epsilon}_{\varkappa}$ the operators of pointwise multiplication by the functions $\epsilon_{\varkappa}: z \mapsto \epsilon_{\varkappa}(z)$ and $\widetilde{\epsilon_{\varkappa}}: z \mapsto \epsilon_{\varkappa}(-z)$ of $z \in \mathbb{R}$ respectively. Both these operators are unitary in the Hilbert space $L_{\mathfrak{h}}^{2}(\mathbb{R})$. If $\hbar \hat{\gamma}=\hat{h}$ is an operator in $L_{\mathfrak{h}}^{2}(\mathbb{R})$ which is given as a pseudo-differential operator $h\left(z, \frac{\hbar}{i} \partial_{z}\right)=\hbar \gamma\left(z, i \partial_{z}\right)$, the operator-function

$$
\gamma(z, \kappa+\varkappa)=\epsilon_{\varkappa+\kappa}^{*}(z) \gamma\left(z, i \partial_{z}\right) \epsilon_{\varkappa+\kappa}(z) \equiv \gamma_{\varkappa+\kappa}(z),
$$

defines the symbol $\gamma_{\varkappa}(z, \kappa)=\gamma(z, \varkappa+\kappa)$ of the operator

$$
\hat{\gamma}_{\varkappa}=\hat{\epsilon}_{\varkappa}^{*} \hat{\gamma} \hat{\epsilon}_{\varkappa} \equiv \gamma_{\varkappa}\left(z, i \partial_{z}\right) \text {. }
$$

It is discribed on the exponential functions $\epsilon_{\kappa}(z)=e^{-i \kappa z}$ as the pseudo-differential operator

$$
\left[\hat{\gamma}_{\varkappa} \epsilon_{\kappa} \eta\right](z)=\gamma_{\varkappa}\left(z, i \partial_{z}\right) e^{-i \kappa z} \eta=e^{-i \kappa z} \gamma_{\varkappa}(z, \kappa) \eta, \eta \in \mathfrak{h} .
$$


Proposition 2. Let $\mathcal{E}_{0}^{-}=\mathcal{E}^{-}$be the Hardy class of $L_{\mathfrak{h}}^{2}(\mathbb{R}), \mathcal{E}_{\varkappa}^{-} \subset L_{\mathfrak{h}}^{2}(\mathbb{R})$ be the Hilbert space of functions $\varphi=\hat{\epsilon}_{\varkappa}^{*} \varphi_{0}$ with $\varphi_{0} \in \mathcal{E}_{0}^{-}$, and $\mathcal{E}_{\varkappa}^{+}=\check{\epsilon}_{\varkappa}^{*} \mathcal{E}_{0}^{+}$, where $\mathcal{E}_{0}^{+}=\mathcal{E}^{+}$. Let the initial boundary-value Schrödinger problem

$$
\begin{array}{ll}
i \partial_{t} \varphi^{t}(z)=\varepsilon_{\varkappa}\left(i \partial_{z}\right) \varphi^{t}(z), & \varphi^{0} \in \mathcal{E}_{\varkappa}^{-}, z>0, \\
i \partial_{t} \tilde{\varphi}^{t}(z)=\tilde{\varepsilon}_{\varkappa}\left(i \partial_{z}\right) \tilde{\varphi}^{t}(z), & \tilde{\varphi}^{t}(0)=\sigma \varphi^{t}(0),
\end{array}
$$

be defined by the generators $\hat{\varepsilon}_{\varkappa}, \check{\varepsilon}_{\varkappa}$ given by the symbols $\varepsilon_{\varkappa}(\kappa)=\varepsilon(\varkappa+\kappa), \tilde{\varepsilon}_{\varkappa}(\kappa)=$ $\varepsilon(\varkappa-\kappa)$ respectively, where $\varepsilon(\kappa)$ is the symmetric function of $\kappa \in \mathbb{R}$, corresponding to the kinetic energy $e(p)=\hbar \varepsilon\left(\hbar^{-1} p\right)>0$. Then it is selfadjoint if the initial output waves $\tilde{\varphi}^{0}$ are defined in $\mathcal{E}_{\varkappa}^{+}$by $\tilde{\varphi}^{0}(-z)=\sigma_{\varkappa}(z) \varphi^{0}(z), z<0$, where $\sigma_{\varkappa}=\epsilon_{\varkappa}^{*} \sigma \epsilon_{\varkappa}$, by analytical continuation of each $\varphi_{0}^{0}=\hat{\epsilon}_{\varkappa} \varphi^{0}$ into the domain $\mathbb{R}^{-}$. The solutions to 3.7) can be written as

$$
\varphi^{t}(z)=\phi^{t}(z), z \geq 0, \tilde{\varphi}^{t}(-z)=\phi^{t}\left(z_{-}\right), z \leq 0
$$

where $\phi^{t}=e^{-i t \hat{\varepsilon}_{\varkappa}} \phi_{t}, \phi_{t}=\varphi^{0}+\left(\hat{\sigma}_{\varkappa}-1\right) \hat{\pi}_{\varkappa}^{t} \varphi^{0}, \hat{\sigma}_{\varkappa}$ is pointwise multiplication by $\sigma_{\varkappa}(z)$, and

$$
\hat{\pi}_{\varkappa}^{t}=e^{i t \hat{\varepsilon}_{\varkappa}} \hat{1}_{0} e^{-i t \hat{\varepsilon}_{\varkappa}} \equiv \pi_{\varkappa}^{t}\left(z, i \partial_{z}\right)
$$

is given by the symbol $\pi^{t}(z, \kappa)$ of the orthoprojector $\hat{\pi}^{t}=e^{i t \hat{\varepsilon}} \hat{1}_{0} e^{-i t \hat{\varepsilon}}$ as in (3.6).

Proof. Separating the variable $t \in \mathbb{R}$ by $\varphi^{t}=e^{-i \varepsilon_{k} t} \varphi_{k}, \tilde{\varphi}^{t}=e^{-i \varepsilon_{k} t} \tilde{\varphi}_{k}$, let us consider the stationary Schrödinger problem

$$
\varepsilon_{\varkappa}\left(z, i \partial_{z}\right) \varphi_{k}(z)=\varepsilon_{k} \varphi_{k}(z), \quad \tilde{\varphi}_{k}(-z)=\sigma_{\varkappa}(z) \varphi_{k}(z)
$$

corresponding to the given initial and boundary conditions in (3.7). Here $\varphi_{k}$ is extended to the domain $\mathbb{R}^{-}$through the analytical continuation of $\epsilon_{\varkappa}^{*} \varphi_{k}$ in $\operatorname{Im} z<0$, which are the generalized eigen-functions (3.2) of $\hat{\varepsilon}=\varepsilon\left(i \partial_{z}\right)$ in $\mathcal{E}_{0}^{-}$iff $k<0$. Due to the self-adjointness of $\hat{\varepsilon}$ in $\mathcal{E}^{-}$, the eigenfunctions $\varphi_{k}=\epsilon_{\varkappa+k}^{*} \eta_{k}$ of $\hat{\varepsilon}_{\varkappa}$ for (3.9) with negative $k$ form an orthocomplete set for the Hilbert space $\mathcal{E}_{\varkappa}^{-}$, and the output eigen-functions $\tilde{\varphi}_{k}(z)=\widetilde{\epsilon_{\varkappa+k}^{*}}(z) \tilde{\eta}_{k}$, where $\tilde{\eta}_{k}=\sigma_{0} \eta_{k}$ with $\sigma_{0}=\rho_{0}^{-1 / 2} \sigma \rho_{0}^{1 / 2}$, form an orthocomplete set for the Hilbert space $\mathcal{E}_{\varkappa}^{+}$. The solutions to (3.7) can be written in the form (3.3) as

$$
\begin{aligned}
& \varphi^{t}(z)=\frac{1}{2 \pi} \int_{-\infty}^{0} e^{-i \varepsilon(k) t} \epsilon_{\varkappa+k}^{*}(z) f(k) \mathrm{d} k=\left[e^{-i t \hat{\varepsilon}_{\varkappa}} \varphi^{0}\right](z), \\
& \widetilde{\varphi}^{t}(z)=\frac{1}{2 \pi} \int_{-\infty}^{0} e^{-i \varepsilon(k) t} \widetilde{\epsilon_{\varkappa+k}^{*}}(z) \tilde{f}(k) \mathrm{d} k=\left[e^{-i t \check{\varepsilon}_{\varkappa}} \widetilde{\varphi}^{0}\right](z),
\end{aligned}
$$

where $\tilde{f}_{0}(k)=\sigma f_{0}(k)$ are defined as the Fourier transforms

$$
f(k)=\int_{-\infty}^{\infty} \epsilon_{\varkappa+k}(z) \varphi^{0}(z) \mathrm{d} z, \tilde{f}(k)=\int_{-\infty}^{\infty} \widetilde{\epsilon_{\varkappa+k}}(z) \tilde{\varphi}^{0}(z) \mathrm{d} z,
$$

by the initial conditions, analytically extended on the whole line $\mathbb{R}$. Due to the commutativity of $\sigma$ and $\hat{\varepsilon}$ they satisfy the connection $\tilde{\varphi}^{t}(-z)=\sigma_{\varkappa}(z) \varphi^{t}(z)$ for all $t$, not only for $t=0$. The time invariance of this connection and the unitarity of the time transformation group in the Hilbert space $\mathcal{E}_{\varkappa}^{-} \oplus \mathcal{E}_{\varkappa}^{+}$, which follows from the unitarity of (3.3) in $\mathcal{E}^{\mp} \subset L_{\mathfrak{h}}^{2}(\mathbb{R})$, means the self-adjointness of the problem (3.7) for the pairs $\varphi^{\mp} \in L_{\mathfrak{h}}^{2}(\mathbb{R}, \boldsymbol{\rho})$ in the domain of the generator $\hat{\varepsilon}_{\varkappa} \oplus \check{\varepsilon}_{\varkappa}$ with the connection $\varphi^{+}(-z)=\sigma_{\varkappa}(z) \varphi^{-}(z)$. Introducing

$$
\phi^{t}(z)=\varphi^{t}(z)+1_{0}(z)\left(\sigma_{\varkappa}(z)-1\right) \varphi^{t}(z)=\sigma_{\varkappa}(z)^{1_{0}(z)} \varphi^{t}(z)
$$


as in (3.5), and taking into account that

$$
\phi^{t}\left(z_{-}\right)=\sigma_{\varkappa}(z)^{1_{0}\left(z_{-}\right)} \varphi^{t}(z)=\sigma_{\varkappa}(z)^{1-1_{0}(-z)} \varphi^{t}(z)=\sigma_{\varkappa}(z)^{-1_{0}(-z)} \tilde{\varphi}^{t}(-z),
$$

we obtain the representation (3.8) as $\varphi^{t}(z)$ coincides with $\phi^{t}(z)$ at $z \geq 0$ and $\tilde{\varphi}^{t}(-z)$ with $\tilde{\phi}^{t}(-z)=\phi^{t}\left(z_{-}\right)$at $z \leq 0$.

Remark 2. The Schrödinger boundary value problem (3.7) is physical in all three aspects. First, the equation (3.7) is invariant under the reversion of time arrow, i.e. under the reflection $t \mapsto-t$ and an isometric complex conjugation $\varphi \mapsto \bar{\varphi}$ together with the input-output exchange $\varphi \leftrightarrows \tilde{\varphi}$ if $\bar{\sigma}=\sigma^{-1}$ and $\bar{\varkappa}=\tilde{\varkappa}$, where $\tilde{\varkappa}(z)=\varkappa(-z)$. Second, the wave functions $\varphi^{t}, \tilde{\varphi}^{t}$ have continuous propagation in both directions of the momentum along $z$, and at the boundary $z=0$ the momentum changes its direction but not the magnitude (conservation of momentum) as the result of the boundary condition $\varphi(0) \mapsto \tilde{\varphi}(0)$. And third, the kinetic energy operator $\hat{\varepsilon}_{\varkappa} \oplus \check{\varepsilon}_{\varkappa}$ is bounded from below as the result of unitary transformation of $\hat{\varepsilon} \simeq \check{\varepsilon}$. 2.4).

Indeed, from $\bar{\varepsilon}(\kappa)=\varepsilon(\kappa)=\tilde{\varepsilon}(\kappa)$ it follows that the symbol $\bar{\varepsilon}_{\varkappa}(z, \kappa)=\overline{\varepsilon_{\varkappa-\kappa}}(z)$ of the complex conjugated operator $\overline{\varepsilon_{\varkappa}}$ is given by

$$
\overline{\varepsilon_{\varkappa}}(z)=\epsilon_{-\bar{\varkappa}}^{*}(z) \varepsilon\left(i \partial_{z}\right) \epsilon_{-\bar{\varkappa}}(z)=\epsilon_{-\tilde{\varkappa}}^{*}(z) \varepsilon\left(i \partial_{z}\right) \epsilon_{-\tilde{\varkappa}}(z)=\widetilde{\varepsilon_{\varkappa}}(z)
$$

if $\bar{\varkappa}=\tilde{\varkappa}$, as $\overline{\epsilon_{\varkappa}}(z)=\epsilon_{-\bar{\varkappa}}(z)$ and $\epsilon_{-\tilde{\varkappa}}(z)=\widetilde{\epsilon_{\varkappa}}(z)$. Therefore $\bar{\varepsilon}_{\varkappa}(z, \kappa)=\tilde{\varepsilon}_{\varkappa}(z, \kappa)$, where $\tilde{\varepsilon}_{\varkappa}(z, \kappa)=\widetilde{\varepsilon_{\varkappa-\kappa}}(z)$ is the symbol for the kinetic energy operator $\check{\varepsilon}_{\varkappa}=\widetilde{\hat{\varepsilon}_{\varkappa}}$ for the output wave $\tilde{\varphi}$. Thus the time reversion with complex conjugation in (3.7) is equivalent to the input-output interchange $\left(\varphi^{t}, \tilde{\varphi}^{t}\right) \mapsto\left(\tilde{\varphi}^{t}, \varphi^{t}\right)$ which preserves the connection between $\varphi^{t}$ and $\tilde{\varphi}^{t}$ as

$$
\overline{\sigma_{\varkappa}}(z)=\epsilon_{-\bar{\varkappa}}^{*}(z) \bar{\sigma} \epsilon_{-\bar{\varkappa}}(z)=\epsilon_{-\tilde{\varkappa}}^{*}(z) \sigma^{-1} \epsilon_{-\tilde{\varkappa}}(z)=\widetilde{\sigma_{\varkappa}}(z)^{-1},
$$

where $\widetilde{\sigma_{\varkappa}}(z)=\sigma_{\varkappa}(-z)$ due to $\bar{\sigma}=\sigma^{-1}$.

\section{The Ultrarelativistic Limit.}

We shall assume here that the symmetric positive kinetic energy $e(p)$ has the relativistic form $|p|$, or more generally, $e(p)=\sqrt{p^{2}+\hbar^{2} \mu^{2}}$ as it was suggested in (3.1). It corresponds to the finite bounds $v_{\mp}=\mp 1$ of the velocity $v(p)=\varepsilon^{\prime}(p)$ at $p \longrightarrow \mp \infty$. Note that the phase speed

$$
\varsigma_{\kappa}=\varepsilon(\kappa) / \kappa=\sqrt{1+\varepsilon^{2} / \kappa^{2}}=\left|v(\hbar \kappa)^{-1}\right|,
$$

for the momenta $p=\mp \hbar \kappa, \kappa>0$ of the harmonic eigen-waves

$$
e^{-i \varepsilon_{\kappa} t} \epsilon_{\kappa}(z)=e^{-i \kappa\left(\varsigma_{\kappa} t+z\right)}, \quad e^{-i \varepsilon_{\kappa} t} \tilde{\epsilon}_{\kappa}(z)=e^{-i \kappa\left(\varsigma_{\kappa} t-z\right)}
$$

has also the limit $\varsigma=1$ at $\kappa \longrightarrow \infty$. Therefore one should expect that the rapidly oscillating input and output waves

$$
\varphi^{t}(z)=e^{-i \kappa(t+z)} \psi^{t}(z), \quad \tilde{\varphi}^{t}(z)=e^{-i \kappa(t-z)} \tilde{\psi}^{t}(z),
$$

in the ultrarelativistic limit $p \longrightarrow \mp \infty$ will propagate as the plane waves with

$$
\psi^{t}(z)=\psi(z+t) \equiv e^{t \partial_{z}} \psi, \quad \tilde{\psi}^{t}(z)=\tilde{\psi}(z-t) \equiv e^{t \tilde{\partial}_{z}} \tilde{\psi}
$$

if the initial conditions are prepared in this form with slowly changing amplitudes $\psi, \tilde{\psi} \in L_{\mathfrak{h}}^{2}(\mathbb{R})$. This propagation will reproduce the boundary-reflection dynamics $\tilde{\psi}^{t}(0)=\sigma \psi(0)$ on the half line $\mathbb{R}^{+} \ni z=s$ if the initial wave amplitudes are 
connected by $\tilde{\psi}(-z)=\sigma \psi(z)$ for all $z \in \mathbb{R}$. In particular, the solutions $\psi^{t}(s)=$ $\psi(s+t), \tilde{\psi}^{t}(s)=0, t<s$,

$$
\tilde{\psi}^{t}(s)=\tilde{\psi}^{t-s}(0)=\sigma \psi^{t-s}(0)=\sigma \psi(t-s), t>s
$$

to this Hamiltonian boundary value problem with the input wave functions

$$
\psi(z)=\sqrt{\rho(z)} \eta, s>0, \quad \psi(z)=0, z \leq 0,
$$

for the initial state-vectors $\eta \in \mathfrak{h}$ will correspond to the single-jump stochastic dynamics in the positive direction of $t$ with respect to the probability density $\rho>0$, $\int_{0}^{\infty} \rho(s) \mathrm{d} s=1$.

Below we give a precise formulation and proof of this conjecture in a more general framework which is needed for the derivation of quantum stochastic evolution as the boundary value problem in second quantization. But first let us introduce the notations and illustrate this limit in this simple case.

In the following we shall use the notion of the inductive limit of an increasing family $\left(\mathcal{E}_{\kappa}\right)_{\kappa>0}$ of Hilbert subspaces $\mathcal{E}_{\kappa} \subseteq \mathcal{E}_{\kappa^{\prime}}, \kappa<\kappa^{\prime}$. It is defined as the union $\mathcal{E}=\cup \mathcal{E}_{\kappa}$ equipped with the inductive convergence which coinsides with the uniform convergence in one of the subspaces $\mathcal{E}_{\kappa}$, and therefore is stronger than the convergence in the uniform completion $\mathcal{K}=\overline{\mathcal{E}}$. The dual inductive convergence is weaker then the convergence in $\mathcal{K}$, and the inductive operator convergence in $\mathcal{E}$ is defined as the operator convergence on each $\mathcal{E}_{\kappa}$ into one of $\mathcal{E}_{\kappa^{\prime}} \subseteq \mathcal{K}$.

Let $\mathcal{G}^{-}=\cup \mathcal{E}_{\kappa}^{-}, \mathcal{G}^{+}=\cup \mathcal{E}_{\kappa}^{+}$be the inductive limits for the increasing family $\left(\mathcal{E}_{\kappa}^{-}, \mathcal{E}_{\kappa}^{+}\right)_{\kappa>0}$ of Hardy classes $\mathcal{E}_{\kappa}^{-}=\hat{\epsilon}_{\kappa}^{*} \mathcal{E}^{-} \supset \mathcal{E}_{\kappa^{\circ}}^{-}, \mathcal{E}_{\kappa}^{+}=\check{\epsilon}_{\kappa}^{*} \mathcal{E}^{+} \supset \mathcal{E}_{\kappa^{\circ}}^{+}, \kappa^{\circ}<\kappa$ in the notations of the previous section. Both $\mathcal{G}^{-}, \mathcal{G}^{+}$are dense in $L_{\mathfrak{h}}^{2}(\mathbb{R})$, consist of the square-intergable $\mathfrak{h}$-valued functions $\psi \in \mathcal{G}^{-}, \tilde{\psi} \in \mathcal{G}^{+}$having zero Fourier amplitudes

$$
g(k)=\int_{-\infty}^{\infty} e^{-i k z} \psi(z) \mathrm{d} z, \quad \tilde{g}(k)=\int_{-\infty}^{\infty} e^{i k z} \tilde{\psi}(z) \mathrm{d} z
$$

for all $k \geq \kappa$ with sufficiently large $\kappa>0$. If $\psi \in \mathcal{E}_{\kappa}^{-}$and $\tilde{\psi} \in \mathcal{E}_{\kappa}^{+}$, then $\varphi=\epsilon_{\kappa} \psi \epsilon$ $\mathcal{E}^{-}, \tilde{\varphi}=\tilde{\epsilon}_{\kappa} \tilde{\psi} \in \mathcal{E}^{+}$, and the free propagation (3.3) can be written in the form (4.1) with

$$
\begin{aligned}
& \psi^{t}=e^{i \kappa t} \hat{\epsilon}_{\kappa}^{*} \varphi^{t}=\hat{\epsilon}_{\kappa}^{*} e^{-i(\hat{\varepsilon}-\kappa 1) t} \hat{\epsilon}_{\kappa} \psi \equiv \psi_{\kappa}^{t}, \\
& \tilde{\psi}^{t}=e^{i \kappa t} \check{\epsilon}_{\kappa}^{*} \tilde{\varphi}^{t}=\check{\epsilon}_{\kappa}^{*} e^{-i(\check{\varepsilon}-\kappa 1) t} \check{\epsilon}_{\kappa} \tilde{\psi} \equiv \tilde{\psi}_{\kappa}^{t} .
\end{aligned}
$$

These unitary transformations in $\mathcal{E}_{\kappa}^{-}$and in $\mathcal{E}_{\kappa}^{+}$, written as

$$
\psi_{\kappa}^{t}(z)=e^{-i t \omega_{\kappa}\left(i \partial_{z}\right)} \psi(z), \quad \tilde{\psi}_{\kappa}^{t}(z)=e^{-i t \tilde{\omega}_{\kappa}\left(i \partial_{z}\right)} \tilde{\psi}(z),
$$

are generated by the selfadjoint operators

$$
\begin{aligned}
& \omega_{\kappa}\left(i \partial_{z}\right)=e^{i \kappa z}\left(\varepsilon\left(i \partial_{z}\right)-\kappa\right) e^{-i \kappa z}=\varepsilon\left(\kappa+i \partial_{z}\right)-\kappa, \\
& \tilde{\omega}_{\kappa}\left(i \partial_{z}\right)=e^{-i \kappa z}\left(\varepsilon\left(i \partial_{z}\right)-\kappa\right) e^{i \kappa z}=\varepsilon\left(\kappa-i \partial_{z}\right)-\kappa
\end{aligned}
$$

They leave all subspaces $\mathcal{E}_{\kappa^{\circ}}^{-}$and $\mathcal{E}_{\kappa^{\circ}}^{+}$invariant respectively, however their generators $\hat{\omega}_{\kappa}, \breve{\omega}_{\kappa}$ are not positive definite for a sufficiently large $\kappa$, and are not unitary equivalent for different $\kappa$ as

$$
\begin{aligned}
& \hat{\epsilon}_{\varkappa} \hat{\omega}_{\kappa} \hat{\epsilon}_{\varkappa}^{*}=\hat{\varepsilon}_{\kappa^{\circ}}-\kappa 1=\hat{\omega}_{\kappa^{\circ}}-\varkappa 1, \\
& \check{\epsilon}_{\varkappa} \check{\omega}_{\kappa} \check{\epsilon}_{\varkappa}^{*}=\check{\varepsilon}_{\kappa^{\circ}}-\kappa 1=\check{\omega}_{\kappa^{\circ}}-\varkappa 1,
\end{aligned}
$$


where $\varkappa=\kappa-\kappa^{\circ}$. Thus we have to prove that the propagation (4.4) has the inductive limit form of plane propagation (4.2) at $\kappa \longrightarrow \infty$ corresponding to the Dirac form of the limits

$$
\lim _{\kappa \rightarrow \infty} \omega_{\kappa}\left(i \partial_{z}\right)=i \partial_{z}, \quad \lim _{\kappa \rightarrow \infty} \tilde{\omega}_{\kappa}\left(i \partial_{z}\right)=-i \partial_{z}
$$

for the Schrödinger generators (4.4).

Another thing which we are going to prove for obtaining the single-jump stochastic limit is that the truncated wave

$$
\chi_{\kappa}^{t}=e^{-i t \hat{\omega}_{\kappa}} \chi_{\kappa, t}, \quad \chi_{\kappa, t}=\psi+(1-\sigma) \hat{\pi}_{\kappa}^{t} \psi
$$

representing the pair (4.4) on the half-line $\mathbb{R}^{+} \ni z$ as in (3.8), has the discontinuous limit

$$
\chi^{t}(z)=\chi_{t}(z+t), \quad \chi_{t}=\psi+(1-\sigma) \hat{1}_{t} \psi .
$$

Here $\hat{1}_{t}=e^{-i t \partial_{z}} \hat{1}_{0} e^{i t \partial_{z}}$ is pointwise multiplication by the characteristic function $1_{t}$ of the interval $-\infty<z<t$ which we shall obtain as the inductive limit of the orthoprojector

$$
\hat{\pi}_{\kappa}^{t}=e^{i t \varepsilon\left(\kappa+i \partial_{z}\right)} \hat{1}_{0} e^{-i t \varepsilon\left(\kappa+i \partial_{z}\right)}=e^{i t \hat{\omega}_{\kappa}} \hat{1}_{0} e^{-i t \hat{\omega}_{\kappa}}
$$

at $\kappa \longrightarrow \infty$. This results are formulated in the following proposition in full generality and notation of the proposition 2 .

Proposition 3. Let $\mathcal{G}^{-}$be the Hilbert inductive limit of Hardy classes $\mathcal{E}_{\kappa}^{-}=\hat{\epsilon}_{\kappa}^{*} \mathcal{E}^{-}$, $\mathcal{G}_{\varkappa}^{-} \subset L_{\mathfrak{h}}^{2}(\mathbb{R}, \rho)$ be the Hilbert space of functions $\psi=\hat{\epsilon}_{\varkappa}^{*} \psi_{0}$ with $\psi_{0} \in \mathcal{G}^{-} \equiv \mathcal{G}_{0}^{-}$, and $\mathcal{G}_{\varkappa}^{+}=\check{\epsilon}_{\varkappa}^{*} \mathcal{G}^{+}$, where $\mathcal{G}^{+}=\cup \mathcal{E}_{\kappa}^{+} \equiv \mathcal{G}_{0}^{+}$. Let the initial boundary-value Schrödinger problem

$$
\begin{aligned}
& i \partial_{t} \psi_{\kappa}^{t}(z)=\omega_{\varkappa, \kappa}\left(i \partial_{z}\right) \psi_{\kappa}^{t}(z), \quad \psi_{\kappa}^{0}=\psi \in \mathcal{G}_{\varkappa}^{-}, z>0, \\
& i \partial_{t} \tilde{\psi}_{\kappa}^{t}(z)=\tilde{\omega}_{\varkappa, \kappa}\left(i \partial_{z}\right) \tilde{\psi}_{\kappa}^{t}(z), z>0, \tilde{\psi}_{\kappa}^{t}(0)=\sigma \tilde{\psi}_{\kappa}^{t}(0),
\end{aligned}
$$

be defined by the generators

$$
\hat{\omega}_{\varkappa, \kappa}=\hat{\epsilon}_{\varkappa}^{*} \hat{\omega}_{\kappa} \hat{\epsilon}_{\varkappa}, \quad \check{\omega}_{\varkappa, \kappa}=\check{\epsilon}_{\varkappa}^{*} \check{\omega}_{\kappa} \check{\epsilon}_{\varkappa}
$$

with the symbols $\omega_{\kappa}, \tilde{\omega}_{\kappa}$ given in (4.4), (3.1), and the initial $\tilde{\psi}_{\kappa}^{0}=\tilde{\psi}$ defined in $\mathcal{G}_{\varkappa}^{+}$ as $\tilde{\psi}(-z)=\sigma_{\varkappa}(z) \psi(z), z<0$ by analytical continuation of each $\psi_{0}=\hat{\epsilon}_{\varkappa} \psi$ into the domain $\mathbb{R}^{-}$. Then the solutions to (4.7) inductively converge to

$$
\psi^{t}(z)=\chi^{t}(z), z \geq 0, \tilde{\psi}^{t}(-z)=\chi^{t}\left(z_{-}\right), z \leq 0
$$

where $\chi^{t}(z)=\epsilon_{\varkappa}(t) \chi_{t}(z+t)$, and $\chi_{t}=\psi+\left(\hat{\sigma}_{\varkappa}-1\right) \hat{1}_{t} \psi$.

Proof. First let us note that the generators in (4.7) have the formal limits

$$
\begin{aligned}
\lim _{\kappa \rightarrow \infty}\left[\hat{\omega}_{\varkappa, \kappa} \psi\right](z) & =\epsilon_{\varkappa}^{*}(z) i \partial_{z}\left[\epsilon_{\varkappa} \psi\right](z)=\left(\varkappa+i \partial_{z}\right) \psi(z), \\
\lim _{\kappa \rightarrow \infty}\left[\check{\omega}_{\varkappa, \kappa} \tilde{\psi}\right](z) & =\widetilde{\epsilon_{\varkappa}^{*}}(z) i \tilde{\partial}_{z}\left[\tilde{\epsilon}_{\varkappa} \tilde{\psi}\right](z)=\left(\varkappa+i \tilde{\partial}_{z}\right) \tilde{\psi}(z)
\end{aligned}
$$

with $\tilde{\partial}_{z}=-\partial_{z}$. This follows from (4.6) and $i \partial_{z} \epsilon_{\varkappa}=\varkappa \epsilon_{\varkappa}, \partial_{z} \widetilde{\epsilon_{\varkappa}}=i \tilde{\varkappa} \widetilde{\epsilon_{\varkappa}}$ as $\widetilde{\epsilon_{\varkappa}}(z)=$ $\epsilon_{-\varkappa}(z)$. Thus we have to prove that the solutions to (4.7) have the limits $\psi=$ 
$\lim \psi_{\kappa}, \tilde{\psi}=\lim \tilde{\psi}_{\kappa}$ in $\mathcal{G}_{\varkappa}^{\mp}$ coinciding with the solutions to the Dirac boundary value problem

$$
\begin{aligned}
& i \partial_{t} \psi^{t}(z)=\left(\varkappa+i \partial_{z}\right) \psi^{t}(z), \quad \psi^{0}=\psi \in \mathcal{G}_{\varkappa}^{-}, z>0 \\
& i \partial_{t} \tilde{\psi}^{t}(z)=\left(\varkappa+i \tilde{\partial}_{z}\right) \tilde{\psi}^{t}(z), z>0, \tilde{\psi}^{t}(0)=\sigma_{0} \psi^{t}(0)
\end{aligned}
$$

with the initial $\tilde{\psi}^{0}$ analytically defined as $\tilde{\psi}^{0}(-z)=\sigma_{\varkappa}(z) \psi^{0}(z)$ in order to keep the solution $\tilde{\psi}^{t}$ also in $\mathcal{G}_{\varkappa}^{-}$for all $t$.

Let us do this using the isomorphisms $\hat{\epsilon}_{\varkappa} \check{\epsilon}_{\varkappa}$ of the dense subspaces $\mathcal{G}_{\varkappa}^{\mp}$ and $\mathcal{G}_{0}^{\mp} \subset L_{\mathfrak{h}}^{2}(\mathbb{R})$. Due this the boundary value problem (4.7) is equivalent to

$$
\begin{aligned}
& i \partial_{t} \psi_{0, \kappa}^{t}(z)=\omega_{\kappa}\left(i \partial_{z}\right) \psi_{0, \kappa}^{t}(z), \quad \psi_{0, \kappa}^{0}=\psi_{0} \in \mathcal{G}_{0}^{-}, z>0 \\
& i \partial_{t} \tilde{\psi}_{0, \kappa}^{t}(z)=\tilde{\omega}_{\kappa}\left(i \partial_{z}\right) \tilde{\psi}_{0, \kappa}^{t}(z), z>0, \tilde{\psi}_{0, \kappa}^{t}(0)=\sigma_{0} \tilde{\psi}_{0, \kappa}^{t}(0),
\end{aligned}
$$

with $\omega_{\kappa}(-k)=\varepsilon(\kappa-k)-\kappa=\tilde{\omega}_{\kappa}(k)$, and $\tilde{\psi}_{0, \kappa}^{0}(-z)=\sigma \psi_{0}(z)$ as $\sigma_{\kappa}=\epsilon_{\kappa}^{*} \sigma \epsilon_{\kappa}=\sigma$ for any scalar $\kappa$. Thus we are to find the ultrarelativistic limit of the solutions

$$
\begin{aligned}
& {\left[e^{-i t \hat{\omega}_{\kappa}} \psi_{0}\right](z)=\frac{1}{2 \pi} \int_{-\infty}^{\kappa} e^{-i\left(t \omega_{\kappa}(-k)-k z\right)} g(k) \mathrm{d} k,} \\
& {\left[e^{-i t \check{\omega}_{\kappa}} \tilde{\psi}_{0}\right](z)=\frac{1}{2 \pi} \int_{-\infty}^{\kappa} e^{-i\left(t \omega_{\kappa}(-k)+k z\right)} \tilde{g}(k) \mathrm{d} k,}
\end{aligned}
$$

with $\tilde{g}(k)=\sigma g(k)$ at $\kappa \longrightarrow \infty$. Here the Fourier amplitudes

$$
g(k)=\int_{-\infty}^{\infty} e^{-i k z} \psi_{0}(z) \mathrm{d} z, \quad \tilde{g}(k)=\int_{-\infty}^{\infty} e^{i k z} \tilde{\psi}_{0}(z) \mathrm{d} z,
$$

are defined by analytical continuation of the initial conditions $\psi_{0} \in \hat{\epsilon}_{\kappa^{\circ}}^{*} \mathcal{E}_{0}^{-}, \tilde{\psi}_{0} \in$ $\breve{\epsilon}_{\kappa^{\circ}}^{*} \mathcal{E}_{0}^{+}$for a $\kappa^{\circ}<\kappa$ such that the integration in (4.9) can be restricted to $k<\kappa^{\circ}$ due to $g(k)=0=\tilde{g}(k)$ for all $k \geq \kappa^{\circ}$. Therefore the proof that the unitary evolution (4.9) inductively converges to the plane propagation $e^{t \partial_{z}} \psi_{0}, e^{t \tilde{\partial}_{z}} \tilde{\psi}_{0}$ resolving this problem at $\kappa \longrightarrow \infty$ can be reduced to finding an estimate of the integral

$$
I\left(\kappa^{\circ}, \kappa\right)=\frac{1}{2 \pi} \int_{-\infty}^{\kappa^{\circ}}\left\|\left(e^{-i\left(k+\omega_{\kappa}(-k)\right) t}-1\right) g(k)\right\|^{2} \mathrm{~d} k .
$$

It gives the value to the mean square distances

$$
\left\|e^{-t \partial_{z}} \psi_{0, \kappa}^{t}-\psi_{0}\right\|^{2}=I\left(\kappa^{\circ}, \kappa\right)=\left\|e^{-t \tilde{\partial}_{z}} \tilde{\psi}_{0, \kappa}^{t}-\tilde{\psi}_{0}\right\|^{2}
$$

of $\psi_{0, \kappa}^{t}(z-t)$ from $\psi_{0} \in \hat{\epsilon}_{\kappa^{\circ}}^{*} \mathcal{E}_{0}^{-}$and of $\tilde{\psi}_{0, \kappa}^{t}(z+t)$ from $\tilde{\psi}_{0} \in \check{\epsilon}_{\kappa^{\circ}}^{*} \mathcal{E}_{0}^{+}$.

To this end we shall use the inequality

$$
\left(\varkappa^{2}+\mu^{2}\right)^{1 / 2}-\varkappa<\frac{1}{2} \frac{\mu^{2}}{\varkappa}, \quad \forall \varkappa>|\mu|
$$

for the monotonously increasing function $k+\omega_{\kappa}(-k)<\kappa^{\circ}+\omega_{\kappa}\left(-\kappa^{\circ}\right)$ of $k<\kappa^{\circ}$. We shall treat separately the three cases in (3.1): the scalar massless case $\mu_{0}=0$ when $\varepsilon(k)=|k|$, the boundedness case $|\mu| \leq m$ when $\varepsilon(k) \leq \sqrt{k^{2}+m^{2}}$ as in the scalar case with $\mu=\mu_{0}>0$, and the general vector case when $\varepsilon(k)=\left(k^{2}+\mu_{0}^{2}-\nabla^{2}\right)^{1 / 2}$.

In the first case $k+\omega_{\kappa}(-k)=k-\kappa+|\kappa-k|=0$ for all $\kappa \geq 0$ and $k<\kappa$. Thus the plane wave propagation

$$
\psi_{0, \kappa}^{t}(z)=\psi_{0}(z+t), \quad \tilde{\psi}_{0, \kappa}^{t}(z)=\tilde{\psi}_{0}(z-t)
$$


is extended by ultrarelativistic limit $\kappa \longrightarrow \infty$ from the orthogonal Hardy classes $\mathcal{E}_{0}^{\mp}$ onto the inductive spaces $\mathcal{G}_{0}^{\mp}$. By continuity they are uniquely defined as the opposite plane propagations on the whole Hilbert space $L_{\mathfrak{h}}^{2}(\mathbb{R})$ where they satisfy the connection $\tilde{\psi}_{0}(-z)=\sigma \psi_{0}(z)$.

In the second case $k+\omega_{\kappa}(-k) \leq m^{2} / 2 \varkappa$ for all $\varkappa=\kappa-\kappa^{\circ}>|\mu|$ and $k<\kappa^{\circ}$. Using the inequality $\left|e^{x}-1\right|<2|x|$ for any $x \in \mathbb{C}$ with $|x| \leq 1$ we obtain the estimate

$$
\left\|I\left(\kappa^{\circ}, \kappa\right)\right\| \leq\left\|e^{-i\left(k+\omega_{\kappa}(-k)\right) t}-1\right\|<2|t|\left\|k+\omega_{\kappa}(-k)\right\|<|t| \frac{m^{2}}{\varkappa}
$$

for the integral $I\left(\kappa^{\circ}, \kappa\right)$ with $\|g\|^{2}=\frac{1}{2 \pi} \int\|g(k)\|^{2} \mathrm{~d} k \leq 1$. Hence for any $\kappa^{\circ}>0$, $\varepsilon>0$ and each $t \in \mathbb{R}$ there exists a $\kappa^{\prime}<\infty$ such that $\left\|I\left(\kappa^{\circ}, \kappa\right)\right\|<\varepsilon$ for all $\kappa>\kappa^{\prime}$. Namely, one can take $\kappa^{\prime}=\kappa^{\circ}+\max \left\{m,|t| m^{2} / \varepsilon\right\}$ such that $\varkappa=\kappa-\kappa^{\circ}>\kappa^{\prime}-\kappa>m$ and $|t| m^{2} / \varkappa<\varepsilon$. Thus the plane wave propagation is indeed the ultrarelativistic limit of (4.9) in the inductive uniform sense.

In the third case one should replace $\mathfrak{h}=L^{2}\left(\mathbb{R}^{d}\right)$ by the inductive limit $\mathfrak{h}^{\circ}=$ $\cup \mathfrak{h}_{\kappa}$ of Hilbert subspaces $\mathfrak{h}_{\kappa}$ of functions in $L^{2}\left(\mathbb{R}^{d}\right)$ having the localized Fourier amplitudes $h(\mathbf{k})=0, \mathbf{k} \notin(-\boldsymbol{\kappa}, \boldsymbol{\kappa})$ for a $\boldsymbol{\kappa} \in \mathbb{R}^{d}$. Then $\mu_{0}^{2}-\nabla^{2}<\mu_{0}^{2}+\boldsymbol{\kappa}^{2}$ in each $\mathfrak{h}_{\kappa}$, and $\left\|I\left(\kappa^{\circ}, \kappa\right)\right\|<|t|\left(\mu_{0}^{2}+\kappa^{2}\right) / \varkappa$ if $\|g\| \leq 1$ for the Fourier amplitudes of $\psi_{0} \in \mathfrak{h}_{\boldsymbol{\kappa}} \otimes \mathcal{E}_{\kappa^{\circ}}^{-}$and of $\tilde{\psi}_{0} \in \mathfrak{h}_{\kappa} \otimes \mathcal{E}_{\kappa^{\circ}}^{+}$, where $\mathcal{E}_{\kappa}^{\mp}$ are Hardy classes in $L^{2}(\mathbb{R})$. Hence for any $\kappa^{\circ}>0, \kappa \in \mathbb{R}^{d}, \varepsilon>0$ and each $t \in \mathbb{R}$ there exists a $\kappa^{\prime}<\infty$ such that $\left\|I\left(\kappa^{\circ}, \kappa\right)\right\|<\varepsilon$ for all $\kappa>\kappa^{\prime}$, namely

$$
\kappa^{\prime}=\kappa^{\circ}+\max \left\{\sqrt{\mu_{0}^{2}+\kappa^{2}},|t|\left(\mu_{0}^{2}+\kappa^{2}\right) / \varepsilon\right\} .
$$

However the estimate $|t|\left(\mu_{0}^{2}+\boldsymbol{\kappa}^{2}\right) /\left(\kappa-\kappa^{\circ}\right)$ depends now on $\boldsymbol{\kappa}$ defining the choice of $g(k)$ in $\mathfrak{h}^{\circ}$ for each $k<\kappa^{\circ}$. This proves that the plane wave propagation is the ultrarelativistic limit of (4.9) also in the general vector case, although not in the uniform but in the strong inductive convergence sense.

Thus the boundary value problem (4.7) in the ultrarelativistic limit is unitary equivalent to the plane propagations (4.2) of opposite waves $\psi_{0}, \tilde{\psi}_{0}$ with the connection $\tilde{\psi}_{0}(-z)=\sigma \psi_{0}(z)$ for all $z \in \mathbb{R}$. In the half space $z \in \mathbb{R}^{+}$this obviously can be written as

$$
\psi_{0}^{t}(z)=\chi_{0}^{t}(z), z \geq 0, \quad \tilde{\psi}_{0}^{t}(-z)=\chi_{0}^{t}\left(z_{-}\right), z \leq 0,
$$

where $\chi_{0}^{t}(z)=\chi_{0, t}(z+t)$ is the truncated input wave (4.5) with $\psi_{0}$ in the capacity of $\psi$. Returning back to $\psi^{t}=\hat{\epsilon}_{\varkappa}^{*} \psi_{0}^{t}$ and $\tilde{\psi}^{t}=\check{\epsilon}_{\varkappa}^{*} \tilde{\psi}_{0}^{t}$ we shall obtain the representation (4.8) with

$$
\chi^{t}(z)=\epsilon_{\varkappa}^{*}(z) e^{t \partial_{z}} \epsilon_{\varkappa}(z) \chi_{t}(z)=\epsilon_{\varkappa}(t) \chi_{t}(z+t),
$$

due to continuity of $\epsilon_{\varkappa}(t)$, where $\chi_{t}=\hat{\epsilon}_{\varkappa}^{*} \chi_{0, t}$ is given in (4.8).

Remark 3. The truncated wave $\chi^{t}=\hat{1}_{0}^{\perp} \psi^{t}+\hat{1}_{0} \tilde{\psi}^{t}$ in the interaction representation $\chi(t)=e^{i \hat{\gamma} t} \chi^{t}$ with respect to the shift group generated by $\hat{\gamma}=i \partial_{z}$ satisfies the stochastic single-jump equation

$$
\mathrm{d} \chi(t, z)+i \varkappa \chi(t, z) \mathrm{d} t=(\sigma-1) \chi(t, z) \mathrm{d} 1_{t}(z), t>0 .
$$


Indeed, the dynamical group $\epsilon_{\varkappa}(t)=e^{-i \varkappa t}$ is unitary in $\mathfrak{h}$. The one-parametric group $e^{t \partial_{z}}$ is apparently generated by the self-adjoint operator $\gamma\left(i \partial_{z}\right)=i \partial_{z}$ in $L^{2}(\mathbb{R})$ which is the symbol of the generator $\hat{\gamma}$ for the shift group evolution $e^{-i t \hat{\gamma}}$. It is a unitary group in $L_{\mathfrak{h}}^{2}(\mathbb{R})$ due to the shift-invariance of the Lebesgue measure on $\mathbb{R}$. Hence the truncated wave in the interaction representation is given by

$$
\begin{aligned}
\chi(t, z) & =\chi^{t}(z-t)=\epsilon_{\varkappa}(t) \chi_{t}(z) \\
& =\epsilon_{\varkappa}^{*}(z-t) \epsilon_{\varkappa}(z) \chi_{t}(z)=e^{i(z-t) \varkappa} \chi_{0, t}(z),
\end{aligned}
$$

where $\chi_{0, t}=\chi_{0}+(\sigma-1)\left(1_{t}-1_{0}\right) \chi_{0}$ with $\chi_{0}=\hat{1}_{0}^{\perp} \psi_{0}+\hat{1}_{0} \sigma \psi_{0}$. Taking into account that $\mathrm{d} t \mathrm{~d} 1_{t}(z)=0$ in the Hilbert space sense as it is zero almost everywhere due to $\mathrm{d} 1_{t}(z)=1 \gg \mathrm{d} t \neq 0$ only for the single point $z=t$ having zero measure, we obtain

$$
\begin{aligned}
\mathrm{d} \chi(t, z) & =e^{i(z-t) \varkappa}\left[(\sigma-1) \mathrm{d} 1_{t}(z) \chi_{0}(z)-i \varkappa \chi_{0, t}(z) \mathrm{d} t\right] \\
& =\left[(\sigma-1) \mathrm{d} 1_{t}(z)-i \varkappa \mathrm{d} t\right] e^{i(z-t) \varkappa} \chi_{0, t}(z) \\
& =\left[(\sigma-1) \mathrm{d} 1_{t}(z)-i \varkappa \mathrm{d} t\right] \chi(t, z) .
\end{aligned}
$$

Here we used that $\mathrm{d} 1_{t}(z)=\mathrm{d} 1_{0}(z-t)=0$ if $z \neq t$ such that

$$
\mathrm{d} 1_{t}(z) e^{i(z-t) \varkappa} \chi_{0, t}(z)=\mathrm{d} 1_{t}(z) \chi_{0, t}(z)=\mathrm{d} 1_{t}(z) \chi_{0, z}(z)
$$

due to $\left.\chi_{0, t}(z)\right|_{t=z}=\chi_{0}(z)$ as $1_{t}(z)-1_{0}(z)=0$ for any $z \geq t \geq 0$. Thus we have proved that $\chi(t, z)$ indeed satisfies the stochastic single jump equation (4.10) in the Hilbert space $L_{\mathfrak{h}}^{2}(\mathbb{R}, \rho)$ of the initial conditions $\chi=\hat{1}_{0}^{\perp} \psi+\hat{1}_{0} \tilde{\psi}$ with respect to the unitary group evolution $e^{t \partial_{z}}$.

Returning to the notations $\varkappa=\hbar^{-1} H, \sigma=S$ of the Sec. 1 in the Hilbert space $\mathfrak{h}=\mathcal{H}$ we obtain the stochastic equation (2.2) for the unitary cocycle $V(t, s)=e^{-t \partial_{z}} V^{t}$, where $V^{t}=S^{\hat{1}_{0}} e^{t\left(\partial_{z}-i \hbar^{-1} H\right)} S^{-\hat{1}_{0}}$, as a quantum-mechanical stochastic approximation. Namely, the toy Hamiltonian model for the interpretation of discontinuous stochastic evolution in terms of the strongly continuous unitary group resolving the Dirac boundary value problem in extra dimension, is indeed the ultrarelativistic inductive limit of a Schrödinger boundary-value problem with bounded from below Hamiltonian $H_{\kappa}(p)=\hbar \omega_{\kappa, \varkappa}\left(-\hbar^{-1} p\right)$.

\section{REFERENCES}

[1] S. Albeverio, V. N. Kolokoltsov, O. G. Smolyanov, "Continuous Quantum Measurement: Local and Global Approaches," Reviews in Mathematical Physics, 9, No. 8, 907-920 (1997)

[2] V. P. Belavkin, "A Dynamical Theory of Quantum Measurement and Spontaneous Localization," Russian Journal of Mathematical Physics, 3, No. 1, 3-23 (1995)

[3] L., Accardi, R., Alicki, A. Frigerio, and Y. G. Lu, "An invitation to the weak coupling and low density limits," Quantum Probability and Related Topics VI, 3-61 (1991)

[4] V. P. Belavkin, "A stochastic Hamiltonian approach for quantum jumps, spontaneous localizations, and continuous trajectories," Quantum Semicalss. Opt. 8, 167-187 (1996)

[5] V. P. Belavkin, "Nondemolition Principle of Quantum Measurement Theory," Foundations of Physics, 24, No. 5, 685-714 (1994)

[6] A. M. Chebotarev, "The quantum stochastic equation is equivalent to a symmetric boundary value problem for the Schrödinger Equation," Mathematical Notes, 61, No. 4, 510-518 (1997)

[7] R. L. Hudson, and K. R. Parthasarathy, "Quantum Ito's formula and stochastic evolutions," Comm. Math. Phys. 93, No. 3, 301-323 (1984)

School of Mathematics, Nottingham University, NG7 2RD, UK

E-mail address: vpb@maths, nott.ac.uk

$U R L$ : www.maths.nott.ac.uk/personal/vpb/ 